\title{
HIGHLY CONNECTED POINCARÉ COMPLEXES
}

\author{
Dedicated to Professor A. Komatu on his 70th birthday
}

\author{
By Seiya Sasao and Hideo Takahashi
}

\section{Introduction.}

We are interested in the following problem proposed by Wall in [2] "Classify up to homotopy $(n-1)$-connected Poincaré complexes of dimension $2 n+1$ and $2 n+2 . "$

In this paper we shall discuss the case of dimension $2 n+2$ under some additional conditions. Let $K$ be a Poincaré complex which is $(n-1)$-connected and of dimension $2 n+2$. If $K$ has the same rational homology as the sphere, then the homology $H_{*}(K ; Z)$ is as follows

$$
\begin{aligned}
& H_{0}(K ; Z)=Z=H_{2 n+2}(K ; Z) \\
& H_{n}(K ; Z)=G=H_{n+1}(K ; Z) \\
& H_{i}(K ; Z)=0 \quad \text { for other dimensions, }
\end{aligned}
$$

where $G$ denotes a finite abelian group. We denote by $P(n, n+1 ; G)$ the complex $K$ such as above and call it a Poincaré complex of type $(n, n+1 ; G)$. Then our main results are

Theorem A. Let $n \geqq 3$ and $G \otimes Z_{2}=0$. Then $P(n, n+1 ; G)$ has the same homotopy type as the connected sum of $P\left(n, n+1 ; G_{1}\right)$ and $P\left(n, n+1 ; G_{2}\right)$ if $G$ is a direct sum of $G_{1}$ and $G_{2}$.

THEOREM B. Under the same conditions as Theorem A, if $P(n, n+1 ; G)$ is S-reducible it's homotopy type is unique with respect to $n$ and $G$.

By applying these theorems to the case of manifolds we shall prove

THEOREM C. Let $M$ be a $(n-1)$-connected rational homology sphere which is a smooth manifold of dimension $2 n+2$ with no 2-torsion. Then $M$ is uniquely determined up to homotopy by homology for $n \equiv 0,1 \bmod 4$.

The case of $G \otimes Z_{2} \neq 0$ (essencially, $G$ is a 2-group) is more complicated, therefore we shall discuss it in the subsequent paper.

Received October 14, 1977 
The plan of this paper is as follows. First, in $\S 1$, we study the homotopy of Moore spaces and in $\S 2$ characterize Poincaré complexes of type $(n, n+1 ; G)$. In $\S 3$ we shall prove Theorem $\mathrm{A}$ and $\mathrm{B}$, and in $\S 4$ the proof of Theorem $\mathrm{C}$ shall be given. Throughout this paper we assume that groups $G, H, \cdots$ are finite abelian with no 2 -torsion and $n \geqq 3$.

$\S 1$. Homotopy of Moore spaces.

We denote by $M_{G}^{n}$ the Moore space of type $(n, G)$ and by $\#$ the integer $2 n+1$. We first note the following easy

LEMMA 1.1. $\pi_{i}\left(M_{G}^{n}\right)$ is trivial for $i=n+1, n+2$.

Now we define a homomorphism

$$
\mu_{I I}^{G}: \pi_{\#}\left(M_{G}^{n} \vee M_{H}^{n+1}\right) \longrightarrow \operatorname{Hom}(G, H)
$$

by

$$
\begin{aligned}
\mu_{H}^{G}(f)=\mu_{f} \cap: G=H^{n+1}\left(M_{G}^{n} ; Z\right)=H^{n+1}(c(f) ; Z) \longrightarrow \\
H_{n+1}(c(f) ; Z)=H_{n+1}\left(M_{H}^{n+1} ; Z\right)=H,
\end{aligned}
$$

where $c(f)$ denotes the mapping cone for a map $f: S^{\#} \rightarrow M_{G}^{n} \vee M_{H}^{n+1}$ and $\mu_{f}$ is the oriented generator of $H_{2 n+2}(c(f) ; Z)$. Let $h$ be a map $M_{G}^{n} \vee M_{H}^{n+1} \rightarrow M_{G^{\prime}}^{n} \vee M_{H^{\prime}}^{n+1}$. Clearly $h$ is decomposed into the sum of four maps;

$$
h_{1}: M_{G}^{n} \longrightarrow M_{G^{\prime}}^{n}, h_{2}: M_{G}^{n} \longrightarrow M_{H^{\prime}}^{n+1}, h_{3}: M_{H}^{n+1} \longrightarrow M_{G^{\prime}}^{n} \text { and } h_{4}: M_{H}^{n+1} \rightarrow M_{H^{\prime}}^{n+1} .
$$

Then, from the commutative diagram

we obtain

$$
\begin{gathered}
H^{n+1}(c(f) ; Z) \underset{\mu_{f} \cap}{h_{1}^{*} \uparrow} H_{n+1}(c(f) ; Z) \\
H^{n+1}(c(h f) ; Z) \underset{\mu_{4 *}}{\longrightarrow} H_{n+1}(c(h f) ; Z),
\end{gathered}
$$

LEMMA 1.2. (The naturality of $\left.\mu_{H}^{G}\right) \mu_{H^{\prime}}^{G^{\prime}}(h f)=h_{4 *} \mu_{H}^{G}(f) h_{1}^{*}$.

Now we prove

Proposition 1.3. $\pi_{\#}\left(M_{G}^{n} \vee M_{H}^{n+1}\right)=\pi_{\#}\left(M_{G}^{n}\right) \oplus \pi_{\#}\left(M_{H}^{n+1}\right) \oplus \operatorname{Hom}(G, H)$

Proof. The proof follows from the standard isomorphism

$$
\pi_{\#}\left(M_{G}^{n} \vee M_{H}^{n+1}\right)=\pi_{\#}\left(M_{G}^{n}\right) \oplus \pi_{\#}\left(M_{H}^{n+1}\right) \oplus \partial \pi_{\#+1}\left(M_{G}^{n} \times M_{H}^{n+1}, M_{G}^{n} \vee M_{H}^{n+1}\right)
$$

if we can show that the restriction $\mu_{H}^{g}$ on the third factor is an isomorphism. Thus, by using isomorphisms

$$
\pi_{\ddagger+1}\left(M_{G}^{n} \times M_{H}^{n+1}, M_{G}^{n} \vee M_{H}^{n+1}\right)=\pi_{\#+1}\left(M_{G}^{n} \wedge M_{H}^{n+1}\right)=\pi_{\#+1}\left(M_{G \otimes H}^{2 n+1} \vee M_{G * H}^{2 n+2}\right),
$$

where $\wedge$ denotes the smash product, the proof can be reduced to the case of $G=Z_{p \imath}$ and $Z_{p \jmath}$. Let $\alpha$ be the generator of 


$$
\pi_{\mp+1}\left(M_{G}^{n} \times M_{H}^{n+1}, M_{G}^{n} \vee M_{H}^{n+1}\right) \cong H_{\#+1}\left(M_{G}^{n} \times M_{H}^{n+1}, M_{G}^{n} \vee M_{H}^{n+1} ; Z\right) \text {. }
$$

Then there exists a map $\varphi: c(f) \rightarrow M_{G}^{n} \times M_{H}^{n+1}(f=\partial \alpha)$ such that $\varphi_{*}: H_{2 n+2}(c(f), Z)$ $\rightarrow H_{2 n+2}\left(M_{G}^{n} \times M_{H}^{n+1} ; Z\right)$ is surjective and $\varphi \mid M_{G}^{n} \vee M_{H}^{n+1}=$ identity. Consider the commutative diagram

$$
Z_{p^{i}}=H_{\uparrow}^{n+1}(c(f) ; Z) \underset{\mu_{f} \cap}{\longrightarrow} H_{n+1}(c(f) ; Z)=Z_{p \jmath}
$$

Then the proof is obtained from $\varphi_{*}\left(\mu_{f}\right) \cap 1=p^{\jmath-k}(1)(k=\min (\imath, j))$.

Now we investigate the $N$-fold suspension

$$
E^{N}: \pi_{\#}\left(M_{\imath}^{n} \vee M_{\imath}^{n+1}\right) \longrightarrow \pi_{\#+N}\left(M_{\imath}^{n+N} \vee M_{\imath}^{N+1+n}\right) \quad(N \longrightarrow \infty),
$$

where $M_{\imath}^{n}$ denotes $M_{G}^{n}$ for $G=Z_{p \imath}$. First, in the decomposition given by Proposition 1.3 , we can easily obtain

$$
E\left(\operatorname{Hom}\left(Z_{p^{\imath}}, Z_{p^{\imath}}\right)\right)=0 \quad \text { and } \quad E^{-N}(0) \cap \pi_{\#}\left(M_{\imath}^{n+1}\right)=\left[\pi_{n+1}\left(M_{\imath}^{n+1}\right), \pi_{\#+1}\left(M_{\imath}^{n+1}\right)\right],
$$

where $[$,$] denotes the Whitehead product. Next, let M_{2, \infty}^{n}$ be the reduced product for $M_{\imath}^{n}$. Using $\pi_{\#+1}\left(M_{\imath}^{n}, \infty, M_{\imath}^{n}\right)=0$ and the homotopy exact sequence of the pair $\left(M_{\imath, \infty}^{n}, M_{\imath}^{n}\right)$, we have

LEMMA 1.4. $E: \pi_{\#}\left(M_{\imath}^{n}\right) \longrightarrow \pi_{\#+1}\left(M_{\imath}^{n+1}\right)$ is injective.

For the investigation of $E: \pi_{\#+1}\left(M_{\imath}^{n+1}\right) \rightarrow \pi_{\#+2}\left(M_{\imath}^{n+2}\right)$ we define a homomorphism $h_{n}: \pi_{2 n}\left(M_{\imath}^{n}\right) \rightarrow Z_{p^{2}}$ as follows. Let $c(f)=M_{\imath}^{n} \cup e^{\#}$ be the mapping cone for a map $f: S^{2 n} \rightarrow M_{\imath}^{n}$ and let $\alpha, \beta, \gamma$ be generators of $H^{n}\left(c(f) ; Z_{p^{\imath}}\right), H^{n+1}\left(c(f) ; Z_{p^{\imath}}\right)$ and $H^{2 n+1}\left(c(f) ; Z_{p^{\imath}}\right)$ respectively. Then put $\mu f \cap(\alpha \cup \beta)=h_{n}(f)$.

LEMMA 1.5. (1) $h_{n}\left(E \pi_{2 n-1}\left(M_{\imath}^{n-1}\right)\right)=0$

(2) if $n$ is even, $h_{n}$ is trivial

(3) if $n$ is odd, $h_{n}$ is surjective

Proof. (1) follows from the definition of $h_{n}$ and (2) is deduced from applying the Bockstein operator. For (3), consider the boundary homomorphism $\partial$ : $\pi_{2 n+1}\left(M_{\imath, \infty}^{n}, M_{\imath}^{n}\right)=Z_{p^{\imath}} \rightarrow \pi_{2 n}\left(M_{\imath}^{n}\right)$. We assert

$$
h_{n}(\partial(1))=\text { a generator of } Z_{p^{2}} \text {. }
$$

Clearly there exists a map $\phi: c(f) \rightarrow M_{\imath, \infty}^{n}$ such that $\psi \mid M_{\imath}^{n}=$ identity and $\phi_{*}: H_{2 n+1}(c(f) ; Z)=Z \rightarrow H_{2 n+1}\left(M_{\imath, \infty}^{n} ; Z\right)$ is surjective. Then our assertion follows from the cohomologyring structure of $M_{\imath, \infty}^{n}$.

LEMMA 1.6. $E^{2}: \pi_{\#}\left(M_{\imath}^{n}\right) \rightarrow \pi_{\#+2}\left(M_{\imath}^{n+2}\right)$ is injective.

Proof. Consider the diagram 


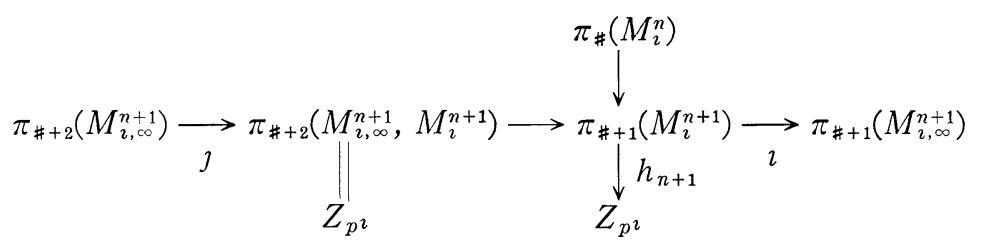

If $n$ is even, the proof follows from lemma 1.4 and (1) of lemma 1.5.

If $n$ is odd, $j$ is surjective by (1) and (3) of lemma 1.5, and hence $i$ is injective. Thus the proof is completed.

Thus, from combining lemmas, we have

PROPOSITION 1.7. The kernel of $E^{N}$ is the subgroup

$$
\left[\pi_{n+1}\left(M_{\imath}^{n+1}\right), \pi_{n+1}\left(M_{\imath}^{n+1}\right)\right] \oplus \operatorname{Hom}\left(Z_{p^{\imath}}, Z_{p^{\imath}}\right) \text {. }
$$

Now let $\iota_{n+1}$ be the generator of $\pi_{n+1}\left(M_{\imath}^{n+1}\right)$ and define the map $\nu_{r}: M_{\imath}^{n} \bigvee$ $M_{\imath}^{n+1} \rightarrow M_{\imath}^{n} \vee M_{\imath}^{n+1}$ by $\nu_{r} \mid M_{\imath}^{n+1}=$ identity and $\nu_{r} \mid M_{\imath}^{n}=$ identity $+r \iota_{n+1}$ id $/ S^{n}$. For the later, we note $+\imath d$.

LEMMA 1.8. For $\imath d \in \operatorname{Hom}\left(Z_{p^{\imath}}, Z_{p^{\imath}}\right) \subset \pi_{\#}\left(M_{\imath}^{n} \vee M_{\imath}^{n+1}\right)$ we have $\nu_{r_{*}}(i d)=r\left[\ell_{n+1}, \iota_{n+1}\right]$

Proof. Since $E^{N}(\imath d)=0$, by Proposition $1.8, \nu_{r_{*}}(\imath d)$ has a representation

$$
\nu_{r_{*}}(\imath d)=x\left[\iota_{n+1}, \iota_{n+1}\right]+y(i d)
$$

for some integers $x$ and $y$. Then $y=1$ follows from the naturality of cupproduct and $x=r$ is easily deduced from the cohomology ring structure of the mapping cone for $\imath d$.

$\S 2$. Poincaré complexes of type $(n, n+1 ; G)$.

First we note

LEMMA 2.1. $P(n, n+1 ; G)$ has the same homotopy type as the mapping cone for a map $f: S^{\#} \rightarrow M_{G}^{n} \vee M_{G}^{n+1}$.

Remark: This is not true in the case of $G \otimes Z_{2} \neq 0$.

Proof. Let $X$ be a Poincaré complex of type $(n, n+1 ; G)$. Since $\pi_{i}(X)=0$ $(0 \leqq i \leqq n-1)$ and $\pi_{n}(X)=G$, we may regard $M_{G}^{n}$ as a subcomplex of $X$. Then we have

$$
\pi_{n+1}(X) \cong \pi_{n+1}\left(X, M_{G}^{n}\right) \cong H_{n+1}\left(X, M_{G}^{n}\right) \cong H_{n+1}(X) \cong G,
$$

using lemma 1.1 and the homotopy-homology exact sequence of the pair $\left(X, M_{G}^{n}\right)$. Hence there is a map $g: M_{G}^{n+1} \rightarrow X$ such that

$$
g_{*}: H_{n+1}\left(M_{G}^{n+1} ; Z\right) \longrightarrow H_{n+1}(X ; Z)
$$


is an isomorphism. Then, since the map $\imath d \vee g: M_{G}^{n} \vee M_{G}^{n+1} \rightarrow X$ induces an isomorphism of homology up to dimension $2 n+1$ the proof is completed by the standard argument.

Thus, from the point of view of homotopy, we can replace a complex of type $(n, n+1 ; G)$ with $c(f)$.

Lemma $2.2 c(f)$ is a Porncaré complex if and only if $f\left(\in \pi_{\#}\left(M_{G}^{n} \vee M_{G}^{n+1}\right)\right)$ is contained in the subgroup

$$
\pi_{\#}\left(M_{G}^{n}\right) \oplus \pi_{\#}\left(M_{G}^{n+1}\right) \oplus \text { Aut } G .
$$

Proof. The part "only if" follows from the definition of decomposition in Proposition 1.3. For the part "if" we must show that two homomorphisms

(1) $\mu_{f} \cap: H^{n+1}(c(f) ; Z) \longrightarrow H_{n+1}(c(f) ; Z)$

(2) $\mu_{f} \cap: H^{n+2}(c(f) ; Z) \longrightarrow H_{n}(c(f) ; Z)$

are both isomorphisms, where $\mu_{f}$ denotes the generator of $H_{2 n+2}(c(f) ; Z)$.

Clearly (1) holds by the definition. Let $Z_{p^{\imath}}, Z_{p}$ be two direct summands of $G$ and let $p_{i}\left(p_{j}\right)$ be the projection $G \rightarrow Z_{p^{\imath}}\left(Z_{p^{j}}\right)$. Since $p_{\imath}$, $p_{\text {, naturally induce }}$ the maps

$$
\hat{p}_{i}: M_{G}^{n} \longrightarrow M_{\imath}^{n} \text { and } \hat{p}_{\jmath}: M_{G}^{n+1} \longrightarrow M_{\jmath}^{n+1} \quad\left(M_{\imath}^{n}=M_{Z_{p}}^{n}\right),
$$

we have the map

$$
\hat{p}_{i} \vee \hat{p}_{j}=p: M_{G}^{n} \vee M_{G}^{n+1} \longrightarrow M_{\imath}^{n} \vee M_{J}^{n+1} .
$$

On the other hand, by lemma 1.2 , we may suppose that $f$ has a representation $f=\alpha \oplus \beta \oplus \imath d$ (Proposition 1.3). Then we have

$$
\begin{aligned}
& p_{*}(f)=\hat{p}_{i^{*}}(\alpha) \oplus \hat{p}_{j^{*}}(\beta) \oplus i d \quad \text { if } \quad Z_{p^{i}}=Z_{p \jmath} \\
& =\hat{p}_{i^{*}}(\alpha) \oplus \hat{p}_{j^{*}}(\beta) \quad \text { if } Z_{p^{i}} \neq Z_{p^{j}},
\end{aligned}
$$

using lemma 1.2. Let $\hat{p}$ be the map $: c(f) \rightarrow c(p f)$ which is the natural extension of $p$ and consider the commutative diagram

$$
\begin{gathered}
G=H^{n+2}(c(f) ; Z) \underset{\hat{p}_{*}}{\uparrow} \underset{\hat{p}_{f} \cap}{\longrightarrow} H_{n}(c(f) ; Z)=G \\
\downarrow \hat{p}_{*}=p_{i} \\
Z_{p \jmath}=H^{n+2}(c(p f) ; Z) \underset{\mu_{p f} \cap}{\longrightarrow} H_{n}(c(p f) ; Z)=Z_{p^{\imath}} .
\end{gathered}
$$

We assert that

$$
\begin{aligned}
& \mu_{p f} \cap Z_{p^{i}}=0 \quad \text { if } \quad Z_{p^{i}} \neq Z_{p j} \\
& =Z_{p^{\imath}} \quad \text { if } \quad Z_{p^{i}}=Z_{p \jmath} .
\end{aligned}
$$

The case of $Z_{p^{\imath}} \neq Z_{p \jmath}$. By (2.4) there exists a map 


$$
q: c(p f) \longrightarrow c\left(\hat{p}_{i} \alpha\right) \vee c\left(\hat{p}_{j} \beta\right)
$$

such that $q \mid M_{2}^{n} \vee M_{j}^{n+1}=\imath d$ and $q_{*}\left(\mu_{p f}\right)=\mu \hat{p}_{i \alpha}+\mu \hat{p}_{j \beta}$. Since $\mu_{\hat{p}_{i \alpha}}$ and $\mu \hat{p}_{j}$ are both trivial we have that $\mu_{p f} \cap$ is also trivial.

The case of $Z_{p \imath}=Z_{p \jmath}$. For our purpose it is sufficient to consider $Z_{p}$-coefficient instead of $Z$-coefficient. Then we can take generators $x\left(\in H^{n}\left(c(p f) ; Z_{p}\right)\right)$ and $y\left(\in H^{n+1}\left(c(p f) ; Z_{p}\right)\right)$ such that $\beta_{\imath} x$ and $\beta_{\jmath} y$ both generators, where $\beta_{\imath}$ denotes the Bockstein operator. Thus, using Kronecker product and (2.3), we have

$$
\begin{aligned}
\left\langle x, \mu_{p f} \cap \beta_{\imath} y\right\rangle & = \pm\left\langle x \cup \beta_{\imath} y, \mu_{p f}\right\rangle= \pm\left\langle\beta_{\imath} x \cup y, \mu_{p f}\right\rangle \\
& = \pm\left\langle y, \mu_{p f} \cap \beta_{\imath} x\right\rangle= \pm 1 .
\end{aligned}
$$

These show our assertion, and therefore the proof of (2) is completed.

3. The proof of Theorem $A$ and $B$.

First we replace a space of type $(n, n+1 ; G)$ with $c(f)$ by lemma 2.1. Let $G=G_{1} \oplus G_{2}$ and let $Z_{v i}(x), Z_{p j}(y)$ be direct summands of $G_{1}$ and $G_{2}$ respectively. By the decomposition

$$
\begin{aligned}
& \pi_{\#}\left(M_{G}^{n} \vee M_{G}^{n+1}\right)=\pi_{\#}\left(M_{G}^{n}\right) \oplus \pi_{\#}\left(M_{G}^{n+1}\right) \oplus \operatorname{Hom}(G, G) \\
= & \pi_{\#}\left(M_{G_{1}}^{n}\right) \oplus \pi_{\#}\left(M_{G_{2}}^{n}\right) \oplus \pi_{\#}\left(M_{G_{1}}^{n+1}\right) \oplus \pi_{\#}\left(M_{G_{2}}^{n+1}\right) \oplus\left[G_{1}, G_{2}\right] \oplus \operatorname{Hom}(G, G),
\end{aligned}
$$

where we identify $G_{\imath}$ with $\pi_{n+1}\left(M_{G_{i}}^{n+1}\right)$, we may suppose that $f$ has the representation

$$
f=\alpha_{1}+\alpha_{2}+\beta_{1}+\beta_{2}+\sum_{x, y} s[x, y]+i d .
$$

For fixed $Z_{p i}\left(x_{0}\right)$ and $Z_{p \jmath}\left(y_{0}\right)$, let $p_{0}$ be the map $M_{G}^{n} \rightarrow M_{2}^{n}$ induced by the projection $G_{1} \rightarrow Z_{p \imath}\left(x_{0}\right)$ and let $p_{0}^{r}$ be the composite map

$$
M_{G_{1}}^{n} \underset{p_{0}}{\longrightarrow} M_{\imath}^{n} \longrightarrow M_{\imath}^{n} / S^{n}=S^{n+1} \underset{r y_{0}}{\longrightarrow} M_{G_{2}}^{n+1} .
$$

Consider the map $F_{r}: M_{G}^{n} \vee M_{G}^{n+1} \rightarrow M_{G}^{n} \vee M_{G}^{n+1}$ defined by $F_{r} \mid M_{G_{1}}^{n+1}=$ identity, $F_{r} \mid M_{G_{2}}^{n+1}=$ identity, $F_{r} \mid M_{G_{2}}^{n}=$ identity and $F_{r} \mid M_{G_{1}}^{n}=$ identity $+p_{0}^{r} . \quad F_{r}$ is clearly a homotopy equivalence and we prove

(1) $F_{r^{*}}\left(\alpha_{2}\right)=\alpha_{2}, \quad F_{r^{*}}\left(\beta_{\imath}\right)=\beta_{\imath} \quad(\imath=1,2)$

(2) $F_{r^{*}}([x, y])=[x, y]$

(3) $F_{r^{*}}\left(\alpha_{1}\right)=\alpha_{1}+p_{0^{*}}^{r}\left(\alpha_{1}\right)$

(4) $F_{r^{*}}(\imath d)=\imath d+r\left[x_{0}, y_{0}\right]$.

For, (1) and (2) are obvious by the definition of $F_{r}$ and (3) follows from $E \pi_{2 n}\left(M_{G_{1}}^{n-1}\right)=\pi_{2 n+1}\left(M_{G_{1}}^{n}\right)$. Since it is easy to obtain

$$
F_{r^{*}}(\imath d)=\imath d+\sum_{x, y} a[x, y]
$$


we must determine a for each $x, y$. Now consider the commutative diagram

$$
\begin{gathered}
M_{G_{1}}^{n} \vee M_{G_{1}}^{n+1} \vee M_{G_{2}}^{n} \vee M_{G_{2}}^{n+1} \\
p_{x}^{n} \vee p_{y}^{n+1} \vee p_{x}^{n} \vee p_{y}^{n+1} \downarrow \\
\quad M_{\imath}^{n} \vee M_{G_{1}}^{n} \vee M_{G_{1}}^{n+1} \vee M_{G_{2}}^{n} \vee M_{G_{2}}^{n+1} \\
\quad M_{\imath}^{n+1} \vee M_{\jmath}^{n} \vee M_{\jmath}^{n+1} \underset{p_{x}^{n+1} \vee p_{y}^{n} \vee p_{y}^{n+1}}{\underset{G_{r}}{\longrightarrow}} M_{\imath}^{n} \vee M_{\imath}^{n+1} \vee M_{\jmath}^{n} \vee M_{\jmath}^{n+1}=X_{x, y},
\end{gathered}
$$

where $G_{r}=\imath d \vee \imath d \vee \imath d \vee \imath d\left((x, y) \neq\left(x_{0}, y_{0}\right)\right), p_{x}^{n}$ is the map $M_{G}^{n} \rightarrow M_{\imath}^{n}$ induced by the projection $G \rightarrow Z_{p i}(x)$, and

$$
G_{r}=\left(\imath d+r y_{0} \circ M_{\imath}^{n} / S^{n}\right) \vee \imath d \vee \imath d \vee \imath d \quad\left((x, y)=\left(x_{0}, y_{0}\right)\right) \text {. }
$$

Then we have

$$
G_{r^{*}}(\imath d)=\imath d+a[x, y] .
$$

Let $\alpha_{x}, \beta_{x}$ be generators for $H^{n+1}\left(M_{\imath}^{n} ; Z_{p k}\right)$ and $H^{n+1}\left(M_{\imath}^{n+1} ; Z_{p k}\right)(k=\mathrm{mim}$ $(\imath, j))$ respectively and we denote by $\hat{X}_{x, y}$ the mapping cone for $\imath d \in \pi_{\#}\left(X_{x, y}\right)$. In the cohomology ring $H^{*}\left(\hat{X}_{x, y} ; Z_{p k}\right)$, we have

$$
\alpha_{x} \cup \beta_{x}=a \text { generator and } \beta_{x} \cup \beta_{y}=0 .
$$

On the other hand, in the cohomology ring $H^{*}\left(c\left(G_{r}(\imath d)\right)\right.$, we have $\beta_{x} \cup \beta_{y}=$ $a(1)$. Hence the proof of (4) follows from

$$
\begin{aligned}
a(1)=G_{r^{*}}\left(\beta_{x}\right) \cup G_{r^{*}}\left(\beta_{y}\right) & =\beta_{x} \cup \beta_{y}=0 \quad\left((x, y) \neq\left(x_{0}, y_{0}\right)\right) \\
& =\beta_{x} \cup\left(r \alpha_{x}+\beta_{y}\right)=r(1) \quad\left((x, y)=\left(x_{0}, y_{0}\right)\right) .
\end{aligned}
$$

Thus the proof of Theorem $\mathrm{A}$ is completed by using iteratedly $F_{r}$ for various $r$

Especially we have

COROllary 3.1. Let $G=\sum_{p} \sum_{i} \sum Z_{p^{2}}$ be the direct-sum decomposition of $G$. Then $P(n, n+1 ; G)$ has the same homotopy type as the connected sum of $P\left(n, n+1 ; Z_{p^{2}}\right)$ s.

Next we consider the proof of Theorem B. Let $G=\sum_{p} \sum_{\imath} \Sigma Z_{p^{2}}$ and let $x$ be

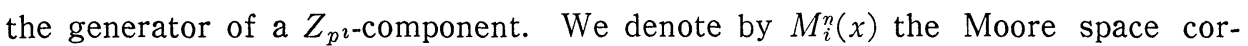
responding to the $Z_{p 2}$-component generated by $x$. By Corollary 3.1 we may assume that $P(n, n+1 ; G)$ has a decomposition

$$
P(n, n+1 ; G)=(\underset{x}{\vee} M(x)) \bigcup_{f} e^{2 n+2}, \quad f=\bigoplus_{x} \sigma_{x} \quad\left(\sigma_{x}=f_{x}+f_{x}^{\prime}+\imath d\right),
$$

where $M(x)$ is the space $M_{i}^{n}(x) \vee M_{i}^{n+1}(x)$ and $\sigma_{x} \in \pi_{\#}(M(x))$. If $P(n, n+1 ; G)$ is $S$-reducible we can know from Proposition 1.7 that

$$
f_{x}=0 \text { and } f_{x}^{\prime} \in\left[\pi_{n+1}\left(M_{2}^{n+1}(x)\right), \pi_{n+1}\left(M_{\imath}^{n+1}(x)\right)\right]
$$

Then, by applying the map $F_{r}$, the proof is completed. 
$\S 4$. $\pi$-manifolds.

We describe a closed smooth manifold as a manifold of type $(n, n+1 ; G)$ if it's underlying Poincaré complex is of type $(n, n+1 ; G)$.

If $M$ is a $\pi$-manifold of type $(n, n+1 ; G), M$ is $S$-reducible and hence it's homotopy type is unique with respect to $n$ and $G$ by Theorem B. Conversely we prove

Proposition 4.1. If $K$ is a S-reducible Poincaré complex of type $(n, n+1 ; G)$, then $K$ has the homotopy type of a $\pi$-manifold.

Proof. Consider the product manifold $S^{n} \times S^{n+2}$ and let $\iota$ be the generator of $\pi_{n}\left(S^{n} \times S^{n+2}\right)$. Since $S^{n} \times S^{n+2}$ is a $\pi$-manifold, a new $\pi$-manifold $K_{m}$ is obtained from killing the class $m \iota$ (Theorem 2 of [1]). Clearly $K_{m}$ is a $\pi$-manifold of type $\left(n, n+1 ; Z_{m}\right)$ and hence it's homotopy type is unique. Then the proof is completed by Theorem B and Corollary 3.1.

Next, for the proof of Theorem C, we prove

Proposition 4.2. Let $n \equiv 0,1 \bmod 4$. Then manıfolds of type $(n, n+1 ; G)$ are all $\pi$-manıfolds.

Proof. Let $M$ be a manifold of type $(n, n+1 ; G)$ and let $\nu_{M}$ be the stable normal bundle for $M$. By lemma 2.1 we may suppose

$$
M=\left(M_{G}^{n} \vee M_{G}^{n+1}\right) \cup e^{2 n+2} \quad \text { (up to homotopy) }
$$

Let $P$ be the natural map $M \rightarrow S^{2 n+2}=M / M_{G}^{n} \vee M_{G}^{n+1}$. Then, from Puppe's sequence, we obtain two isomorphisms

$$
\begin{array}{ll}
P^{*}: Z=\left[S^{2 n+2}, B O\right]_{0} \rightarrow[M, B O]_{0} & (n \equiv 1 \bmod 4) \\
P^{*}: Z_{2}=\left[S^{2 n+2}, B O\right]_{0} \rightarrow[M, B O]_{0} & (n \equiv 0 \bmod 4) .
\end{array}
$$

Thus, there exists a bundle $\xi$ over $S^{2 n+2}$ with $P^{*}(\xi)=\nu_{M}$. Since the Thom space $T\left(\nu_{M}\right)$ is $S$-reducible and $P$ is of degree $1, T(\xi)$ is also reducible, hence we have $J(\xi)=0$. If $n \equiv 1 \bmod 4, J(\xi)=0$ is equivalent to $\xi=0$. Therefore we have $\nu_{M}=p^{*}(\xi)=0$. If $n \equiv 1 \bmod 4, \xi$ is determined by it's Pontrijagin class. Using Hirzeburch formula for $\nu_{M}$ and Index $(M)=0$, we can know that the top Pontrijagin class of $\nu_{M}$ is zero. Thus we get $\xi=0$, i. e. $\nu_{M}=0$.

Now Theorem $\mathrm{C}$ is clear from Proposition 4.2. Finally we note

Proposition 4.3. Let $M$ be an almost parallerizable manifold of type $(n, n+1 ; G)$. Then $M$ is a $\pi$-manifold and hence it's homotopy type is unique with respect to $n$ and $G$.

Proof. Let $\nu_{M}$ be the stable normal bundle for $M$. Since the restriction $\nu_{M} \mid M_{G}^{n} \vee M_{G}^{n+1}$ is trivial, the proof follows from the same argument as the proof of Proposition 4.2. 


\section{REFERENCES}

[1] J.W. MiLnoR, A procedure for killing homotopy groups of differentiable manifolds, Differential Geometry: Symposia in Pure Math, A. M. S. 1961, 39-55.

[2] C. T.C. WaLl, Manifolds-Tokyo 1973, 429-430.

Dept. OF MATH.

Tokyo Inst. of TEChnology.

OH-OKAyama Meguro-KU,

TOKYO, JAPAN. 